\title{
Synthesis and Characterization of a Series of 1-Aryl-4-[aryldiazenyl]- piperazines. Part I. Isomers of N-(2,3-Dimethylphenyl)-N'-(Aryldiazenyl)- Piperazines
}

\author{
Chenguang Fan and Keith Vaughan*
}

Department of Chemistry, Saint Mary’s University, Halifax, Nova Scotia, B3H 3C3, Canada

\begin{abstract}
This paper describes the synthesis of several new series of 1-(2-aryldiazen-1-yl-) 4-arylpiperazines and 1-(2aryldiazen-1-yl-)4-arylalkylpiperazines by using diazonium coupling between arenediazonium ions with the appropriate 1 -arylpiperazine or the 1-arylalkylpiperazine. The new compounds have a common thread in that they are isomers of the series of N-(2,3-dimethylphenyl)- N'-(aryldiazenyl)-piperazines. The new triazenes have been characterized by IR and NMR spectroscopy and mass spectrometry.
\end{abstract}

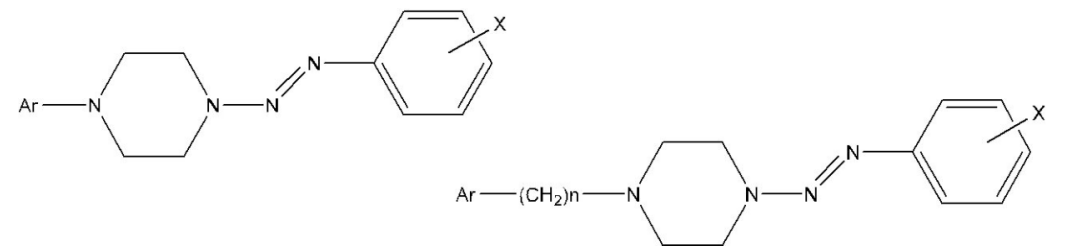

Keywords: Arylpiperazine; diazonium ion, 1-(2,3-dimethylphenyl-)piperazine, IR spectroscopy, Mass spectrometry, 1-(2methylphenylmethyl-)piperazine, 1-(3-methylphenylmethyl-)piperazine, 1-(4-methylphenylmethyl-)piperazine, NMR spectroscopy, piperazine, triazene.

\section{INTRODUCTION}

Previous work in this laboratory has described the synthesis of the 1,4-bis-(2-aryl-diazen-1-yl-)piperazines $(\underline{\mathbf{1}})[1,2]$ using the bis-diazonium coupling reaction with piperazine itself (equation (i)):

[i]

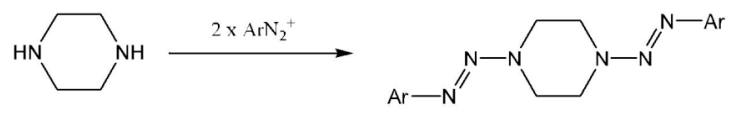

In a similar fashion, diazonium coupling with 1 methylpiperazine afforded a series of 1-(2-aryldiazen-1-yl)-methylpiperazines (ㅁa) [3](equation ii):

[ii]
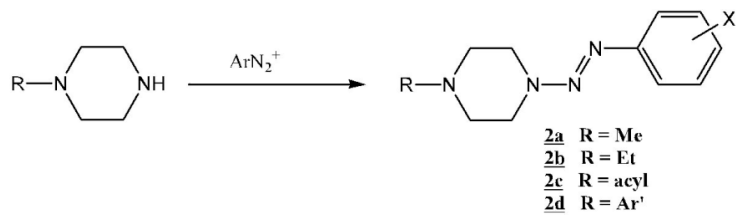

Subsequently, this work was extended to the synthesis of the 1-(2-aryldiazen-1-yl-) 4-ethylpiperazines (므) [4]. Further investigation led to the synthesis of a large number of 1-(2-aryldiazen-1-yl-)4-acylpiperazines (2c); the paper describing these latter results has recently been published [5]. The next logical step in the pursuit of new molecules of

"Address correspondence to this author at the Department of Chemistry, Saint Mary's University, Halifax, Nova Scotia, B3H 3C3, Canada;

Tel: 902-420-5650; E-mail: keith.vaughan@smu.ca the $\mathrm{N}$-aryldiazenylpiperazines is to investigate the synthesis of the1-aryl-4-[aryldiazenyl] -piperazines $(\underline{\mathbf{2 d}})$ by diazonium salt coupling with a series of 1 -arylpiperazines.

In the present work, a series of N-(2,3-dimethylphenyl)N'-(aryl-diazenyl)-piperazines ( $\underline{\boldsymbol{3}})$ have been synthesized by diazonium coupling with $\mathrm{N}$-(2,3-dimethylphenyl-)piperazine. These new triazenes represent the first ever piperazine derivatives to be reported with one N-aryl substituent at N4 opposite to the $\mathrm{N}$-aryldiazenyl substituent at N1. The common thread of all the new compounds reported in this paper is that they are isomers of the compounds of series 3 .

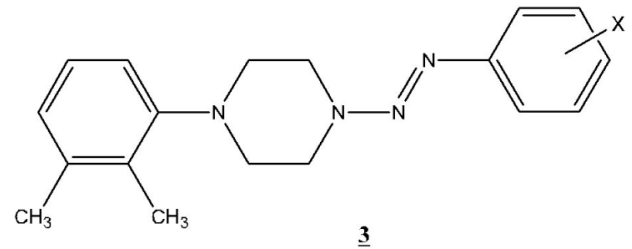

Isomeric compounds described as 1-(methylphenylmethyl)-4-(aryldiazenyl)-piperazines ( $\underline{\mathbf{4}}, \underline{\mathbf{5}}$ and $\underline{\mathbf{6}}$ ) have also been prepared and characterized:

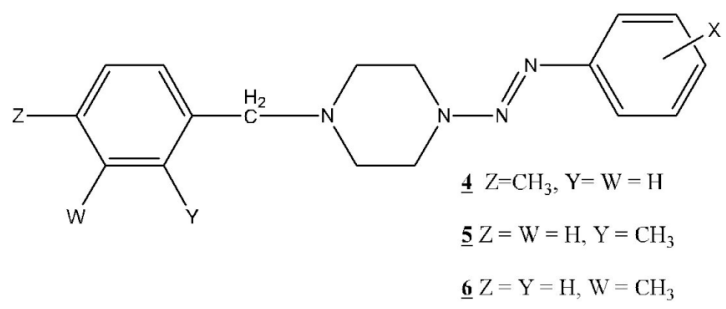


Also the isomer methyl 4-[2-(4-phenethylpiperazino)-1diazenyl]benzoate ( $\mathbf{7})$ has been prepared and characterized.

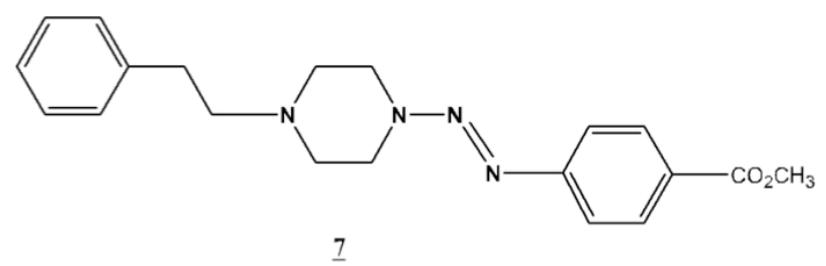

\section{EXPERIMENTAL}

\section{Materials and Apparatus}

The series of aromatic primary amines and the series of 1 -arylsubstituted-piperazines were reagent-grade materials purchased from the Aldrich Chemical Co. Ltd., and were used without further purification. Melting points were determined on a Fisher-Johns Melting Point Apparatus and were uncorrected. Infrared spectra were obtained using nujol mulls on a Bruker Vector-22 IR spectrometer. ${ }^{1} \mathrm{H}$ and ${ }^{13} \mathrm{C}$ NMR spectra were obtained on Bruker AC $250 \mathrm{MHz}$ and $500 \mathrm{MHz}$ spectrometers at the Atlantic Regional Magnetic Resonance Center at Dalhousie University in Halifax, Nova Scotia. Chemical shifts were recorded in $\mathrm{CDCl}_{3}$ solutions at room temperature, and were related to TMS internal standard. The NMR data were interpreted using the TOPSPIN software. The high resolution mass spectra were obtained at Dalhousie University in Halifax, Nova Scotia. Accurate mass measurements were made on a CEC 21-110B mass spectrometer operated at a mass resolution of 8000 ( $10 \%$ valley) by computer-controlled peak matching to appropriate PFK reference ions. Spectra were obtained using electron ionization at 70 volts and a source temperature of $175^{\circ} \mathrm{C}$, with samples being introduced by means of a heatable quartz probe. The standard deviation of mass measurement is $+/-0.0008 \mathrm{amu}$, which is an average of 3.6 ppm over the mass range 100 to $300 \mathrm{amu}$.

\section{General Procedure}

The aromatic primary amine $(0.006 \mathrm{~mol})$ was dissolved in $6 \mathrm{~mL}$ of $3 \mathrm{~mol} / \mathrm{L}$ hydrochloric acid, with the aid of heat if necessary, and the resulting solution cooled in an ice salt bath to below $5{ }^{\circ} \mathrm{C}$. The solution was diazotized with a solution of sodium nitrite $(0.006 \mathrm{~mol})$ in $3 \mathrm{~mL}$ water, with the temperature maintained below $5{ }^{\circ} \mathrm{C}$. Then, the solution was stirred for a further $30 \mathrm{~min}$ in the ice bath. A piperazine solution was prepared by mixing the 1-aryl-piperazine $(0.005$ mol) with $10 \mathrm{~mL}$ water; if necessary, a few drops of a dilute hydrochloric acid solution was added to get the arylpiperazine to dissolve. Then, the piperazine solution was added slowly to the diazonium salt solution. After stirring for an additional $30 \mathrm{~min}$, the mixture was neutralized with a saturated sodium bicarbonate solution and then left to stir until precipitation was deemed to be complete ( $\sim 1$ hour). The solid product was filtered under suction, dried, and recrystallized from an appropriate solvent. Physical data (i.e. yield, m.p., recrystallization solvent) and spectroscopic analysis data (i.e. FT-IR, NMR, and high resolution MS data) were collected.
Synthesis of Series of N-(2,3-dimethylphenyl)-N'(aryldiazenyl)-piperazines (3)

The compounds of series 3a-f were prepared following the general procedure described above. However, during the experiments, there was a problem which may have affected the results. The diazo coupling reaction took place in an aqueous solution. One of the major starting materials, 1-(2,3Xylyl)-piperazine monohydrochloride was not completely dissolved in water at room temperature even when extra hydrochloric acid was added. It could be dissolved in water with heat, but the reaction system had to be cooled all the time as described in the general procedure. Thus, the low solubility of the 1-(2,3-Xylyl)-piperazine may have limited the completion of the reaction. Nevertheless, the 1-(2,3Xylyl)-piperazine monohydrochloride in water may have an equilibrium between the dissolved sample and the undissolved sample, when the dissolved 1-(2,3-Xylyl)piperazine has been used in the reaction, then more of the undissolved piperazine will be dissolved in water. Nevertheless, the longer reaction time may have served to overcome the effect of this factor, as evident in the high yields of compounds of series $\underline{\mathbf{3}}$ reported in Table $\mathbf{1}$.

\section{Synthesis of the Series of 1-(4-methylbenzyl)-4- (aryldiazenyl)-piperazines (4)}

Following the general procedure described above, the experiments were performed in half scale due to the limit of the amount of starting materials. The same substituents of the aromatic amine were applied; the physical and IR data of the final products for this series are given in Table $\mathbf{2}$.

\section{Synthesis of the Series of 1-(2-methylbenzyl)-4- (aryldiazenyl)-piperazines (5)}

The procedure follows the general procedure in the first several steps, until the mixture was neutralized with saturated sodium bicarbonate solution. After the neutralization, a sticky precipitate formed, which was isolated by filtration. The mother-liquor was stored in a fridge. In some cases, a further batch of solid product was isolated from the mother liquor after several days; the second batch was combined with the first batch for the purification. The oily sticky solid was dissolved in dichloromethane and transferred into an Erlenmeyer flask which then was placed in a fume hood for overnight or longer to allow the solvent to evaporate completely. The resulting sticky solid was dissolved in a minimum volume of the appropriate hot solvent (e.g. Ethanol) and then cooled slowly. The crystalline solid was precipitated, then filtered under suction and air dried. Physical data (i.e. yield, m.p., recrystallization solvent) and IR spectroscopic data are given in Table $\mathbf{3}$.

\section{Synthesis of Series of 1-(3-methylbenzyl)-4- (aryldiazenyl)-piperazines (6)}

Following the general procedure described above for series 3, the synthesis of the compounds of series $\mathbf{6}$ were performed in half scale due to the limit of the amount of starting materials. The same substituents of the aromatic 
amine were applied; the physical and IR data of the final products for this series are given in Table 4.

\section{Synthesis of Methyl 4-[2-(4-phenethylpiperazino)-1- diazenyl]benzoate (7)}

The synthetic procedure follows the general procedure described above. Due to the limited amount of the 1-(2phenylethyl)-piperazine available, only one substituted aromatic amine $\left(p-\mathrm{CO}_{2} \mathrm{CH}_{3}\right)$ was applied to the synthesis. The physical data is shown in Table $\mathbf{5}$.

\section{RESULTS AND DISCUSSION}

\section{Synthesis}

The N-aryl-N'-(2-aryl-1-diazenyl-)piperazines ( $\underline{\mathbf{3}}$ to $\underline{\mathbf{7}})$ were synthesized by diazotization of an aromatic primary amine $\left(\mathrm{ArNH}_{2}\right)$ in hydrochloric acid, followed by coupling of the diazonium ion with the appropriate $\mathrm{N}$-arylpiperazine or N-arylalkylpiperazines. Most of the crude products were obtained in good yield $(57-100 \%)$ with the exception of compound 5c. The compounds were purified by recrystallization in high recovery.

\section{Infrared Spectral Analysis}

The significant diagnostic results of the Infrared spectra of the new compounds are shown in Table $\mathbf{1}$ to Table 5. All of the compounds show out-of-plane bending vibration modes of the substituted benzene rings. The paradisubstituted benzene ring, which is present in most of the compounds, except 3f, $\mathbf{4 f}$, and 5e, show out-of-plane bending vibration modes in the range of $825-859 \mathrm{~cm}^{-1}$. Compounds 3f, 4f, and 5e involve a monosubstituted benzene ring, which show two out-of-plane bending vibration modes in the ranges of 693-697 $\mathrm{cm}^{-1}$ and 761-765 $\mathrm{cm}^{-1}$. The $\mathrm{N}$-aryl group,

Table 1. Physical data for the N-(2,3-dimethylphenyl)-N'-(aryl-diazenyl)-piperazines (3).

\begin{tabular}{|c|c|c|c|c|c|c|}
\hline \multirow{2}{*}{ No } & \multirow{2}{*}{$\mathbf{X}$} & Crude & \multirow[t]{2}{*}{$\operatorname{Mp}\left({ }^{\circ} \mathrm{C}\right)$} & \multirow{2}{*}{$\begin{array}{c}\text { Recryst. } \\
\text { Solv. }\end{array}$} & \multirow{2}{*}{$\begin{array}{c}\text { Crystal } \\
\text { Appearance }\end{array}$} & \multirow{2}{*}{$\operatorname{IR}\left(\mathrm{cm}^{-1}\right)$} \\
\hline & & yield & & & & \\
\hline & & $(\%)$ & & & & \\
\hline \multirow[t]{4}{*}{$3 \mathbf{a}$} & $\mathrm{p}-\mathrm{CN}$ & $87.3 \%$ & $147-148$ & Ethanol & Red-brown & $2220(\mathrm{CN})$ \\
\hline & & & & & Needles & 848(para) \\
\hline & & & & & & $785 \& 724$ \\
\hline & & & & & & (1,2,3-trisub) \\
\hline \multirow[t]{5}{*}{$\mathbf{3 b}$} & $\mathrm{p}-\mathrm{CO}_{2} \mathrm{CH}_{3}$ & $63.7 \%$ & $155-157$ & Ethanol & Small orange & $1714(\mathrm{C}=\mathrm{O})$ \\
\hline & & & & & Plates & $1275(\mathrm{C}-\mathrm{O})$ \\
\hline & & & & & & 856(para) \\
\hline & & & & & & $780 \& 724$ \\
\hline & & & & & & (tri-sub) \\
\hline \multirow[t]{3}{*}{$3 c$} & $\mathrm{p}-\mathrm{Br}$ & $100.0 \%$ & $103-103.5$ & Ethanol & Fibrous & 833(para) \\
\hline & & & & & Off-white & $782 \& 719$ \\
\hline & & & & & Plates & (tri-sub) \\
\hline \multirow[t]{3}{*}{ 3d } & $\mathrm{p}-\mathrm{CH}_{3}$ & $81.8 \%$ & $132-132.5$ & Ethanol & Needles & 828(para) \\
\hline & & & & & & $788 \& 723$ \\
\hline & & & & & & (tri-sub) \\
\hline \multirow[t]{5}{*}{$3 e$} & $\mathrm{p}-\mathrm{NO}_{2}$ & $100.0 \%$ & $152-155$ & Ethanol & Red-brown & $1507 \&$ \\
\hline & & & & & Needles & $1341\left(\mathrm{NO}_{2}\right)$ \\
\hline & & & & & & 851(para) \\
\hline & & & & & & $787 \& 721$ \\
\hline & & & & & & (tri-sub) \\
\hline \multirow[t]{3}{*}{$3 \mathbf{f}$} & $\mathrm{H}$ & $57.7 \%$ & $102-102.5$ & Ethanol & Lustrous & $697 \& 763$ \\
\hline & & & & & Gold plates & (mono-sub) \\
\hline & & & & & & $788 \& 724$ \\
\hline
\end{tabular}


Table 2. Physical data for series of 1-(4-methylbenzyl)-4-(aryldiazenyl)-piperazines (4).

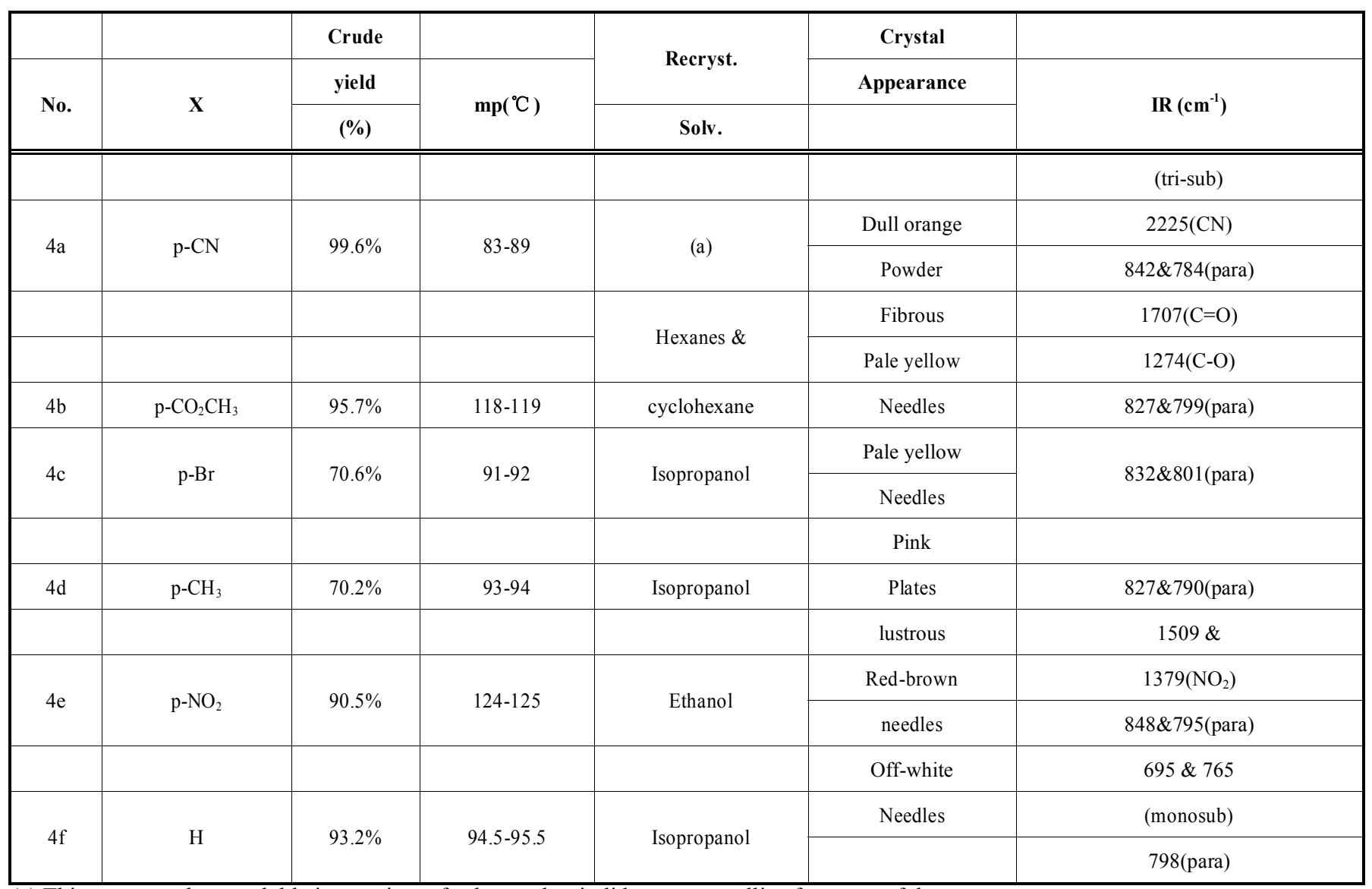

(a) This compound was soluble in a variety of solvents, but it did not recrystallize from any of them.

Table 3. Physical data for series of 1-(2-methylbenzyl)-4-(aryldiazenyl)-piperazines (5).

\begin{tabular}{|c|c|c|c|c|c|c|}
\hline No. & $\mathbf{X}$ & $\begin{array}{c}\text { Overall } \\
\text { yield } \\
(\%)\end{array}$ & $\mathrm{mp}\left({ }^{\circ} \mathrm{C}\right)$ & $\begin{array}{l}\text { Recryst. } \\
\text { Solvent }\end{array}$ & $\begin{array}{c}\text { Crystal } \\
\text { appearance }\end{array}$ & $\operatorname{IR}\left(\mathrm{cm}^{-1}\right)$ \\
\hline $5 \mathrm{a}$ & $\mathrm{p}-\mathrm{CN}$ & $47 \%$ & $73-74$ & $\begin{array}{c}\text { Iso- } \\
\text { Propanol }\end{array}$ & $\begin{array}{l}\text { Off-white } \\
\text { prisms }\end{array}$ & $\begin{array}{l}2218(\mathrm{CN}) \\
840 \text { (para) } \\
745 \text { (ortho) }\end{array}$ \\
\hline $5 b$ & $\mathrm{p}-\mathrm{CO}_{2} \mathrm{CH}_{3}$ & $52 \%$ & $97.5-98.5$ & $\begin{array}{c}\text { Iso- } \\
\text { Propanol }\end{array}$ & $\begin{array}{l}\text { Pale pink } \\
\text { Needles }\end{array}$ & $\begin{array}{c}1714(\mathrm{C}=\mathrm{O}) \\
1274(\mathrm{C}-\mathrm{O}) \\
859 \text { (para) } \\
746 \text { (ortho) }\end{array}$ \\
\hline $5 \mathrm{~d}$ & $\mathrm{p}-\mathrm{NO}_{2}$ & $66.5 \%$ & $92.5-93$ & Ethanol & $\begin{array}{c}\text { Red-brown } \\
\text { Prisms }\end{array}$ & $\begin{array}{c}1509 \& \\
1378\left(\mathrm{NO}_{2}\right) \\
853 \text { (para) } \\
745 \text { (ortho) }\end{array}$ \\
\hline
\end{tabular}


Table 3. contd....

\begin{tabular}{|c|c|c|c|c|c|c|}
\hline No. & $\mathbf{X}$ & $\begin{array}{c}\text { Overall } \\
\text { yield } \\
(\%)\end{array}$ & $\mathrm{mp}\left({ }^{\circ} \mathrm{C}\right)$ & $\begin{array}{c}\text { Recryst. } \\
\text { Solvent }\end{array}$ & $\begin{array}{c}\text { Crystal } \\
\text { appearance }\end{array}$ & IR (cm-1) \\
\hline $5 \mathrm{e}$ & $\mathrm{H}$ & $72 \%$ & $71-73$ & $\begin{array}{c}\text { Hexanes } \\
\text { (a) }\end{array}$ & $\begin{array}{c}\text { Fibrous } \\
\text { Pale yellow } \\
\text { Prisms }\end{array}$ & $\begin{array}{l}693 \& 761 \\
\text { (mono-sub) } \\
743 \text { (ortho) }\end{array}$ \\
\hline
\end{tabular}

(a) By trituration

Table 4. Physical data for series of 1-(3-methylbenzyl)-4-(aryldiazenyl)-piperazines (6).

\begin{tabular}{|c|c|c|c|c|c|c|}
\hline \multirow[b]{2}{*}{ No. } & \multirow[b]{2}{*}{$\mathbf{x}$} & Crude & \multirow[b]{2}{*}{$\operatorname{mp}\left({ }^{\circ} \mathrm{C}\right)$} & Recryst. & Crystal & \multirow[b]{2}{*}{$\operatorname{IR}\left(\mathrm{cm}^{-1}\right)$} \\
\hline & & $(\%)$ & & Solv. & & \\
\hline \multirow[b]{2}{*}{$6 a$} & & & & & Pale orange & $2220(\mathrm{CN})$ \\
\hline & $\mathrm{p}-\mathrm{CN}$ & $90 \%$ & $105-106$ & iso-propanol & Needles & 841 (para) \\
\hline \multirow{3}{*}{$6 \mathrm{~b}$} & & & & & Lustrous & $1710(\mathrm{C}=\mathrm{O})$ \\
\hline & & & & & Flesh-colored & 860 (para) \\
\hline & $\mathrm{p}-\mathrm{CO}_{2} \mathrm{CH}_{3}$ & $93 \%$ & $82-83$ & ethanol & Plates & $775 \& 698$ (meta) \\
\hline $6 c$ & & & & & Tiny buff & \\
\hline \multirow[t]{3}{*}{ 6d } & & & & & Pink & 823 (para) \\
\hline & $\mathrm{p}-\mathrm{CH}_{3}$ & $62 \%$ & $85-86$ & Iso-propanol & Needles & $781 \& 696$ (meta) \\
\hline & & & & & Tiny & $1514 \&$ \\
\hline \multirow{3}{*}{$6 e$} & & & & & Red-brown & $1376\left(\mathrm{NO}_{2}\right)$ \\
\hline & & & & & Needles & 853(para), \\
\hline & $\mathrm{p}-\mathrm{NO}_{2}$ & $94 \%$ & $82-83$ & Iso-propanol & & $781 \& 694(\mathrm{~m})$ \\
\hline
\end{tabular}

Table 5. Physical data for Methyl 4-[2-(4-phenethylpiperazino)-1-diazenyl]benzoate (7).

\begin{tabular}{|c|c|c|c|c|c|c|}
\hline & \multirow[b]{2}{*}{$\mathbf{X}$} & Crude & Mp & Recryst. & Crystal & \multirow[b]{2}{*}{$\operatorname{IR}\left(\mathrm{cm}^{-1}\right)$} \\
\hline No. & & (\%) & $\left({ }^{\circ} \mathrm{C}\right)$ & Solv. & & \\
\hline & & & & & & $1722(\mathrm{C}=\mathrm{O})$ \\
\hline & & & & & & $1278(\mathrm{C}-\mathrm{O})$ \\
\hline & & & & & Creamy white & $699 \& 774$ \\
\hline $7 a$ & $\mathrm{p}-\mathrm{CO}_{2} \mathrm{CH}_{3}$ & $97.1 \%$ & $98-99$ & Ethanol & prisms & (mono-sub) \\
\hline
\end{tabular}


Table 6. ${ }^{1} \mathrm{H}$ NMR Data for compounds 3a-f; chemical shifts in ppm relative to $\mathrm{TMS}(1 \%)$ at room temperature in $\mathrm{CDCl}_{3}$.

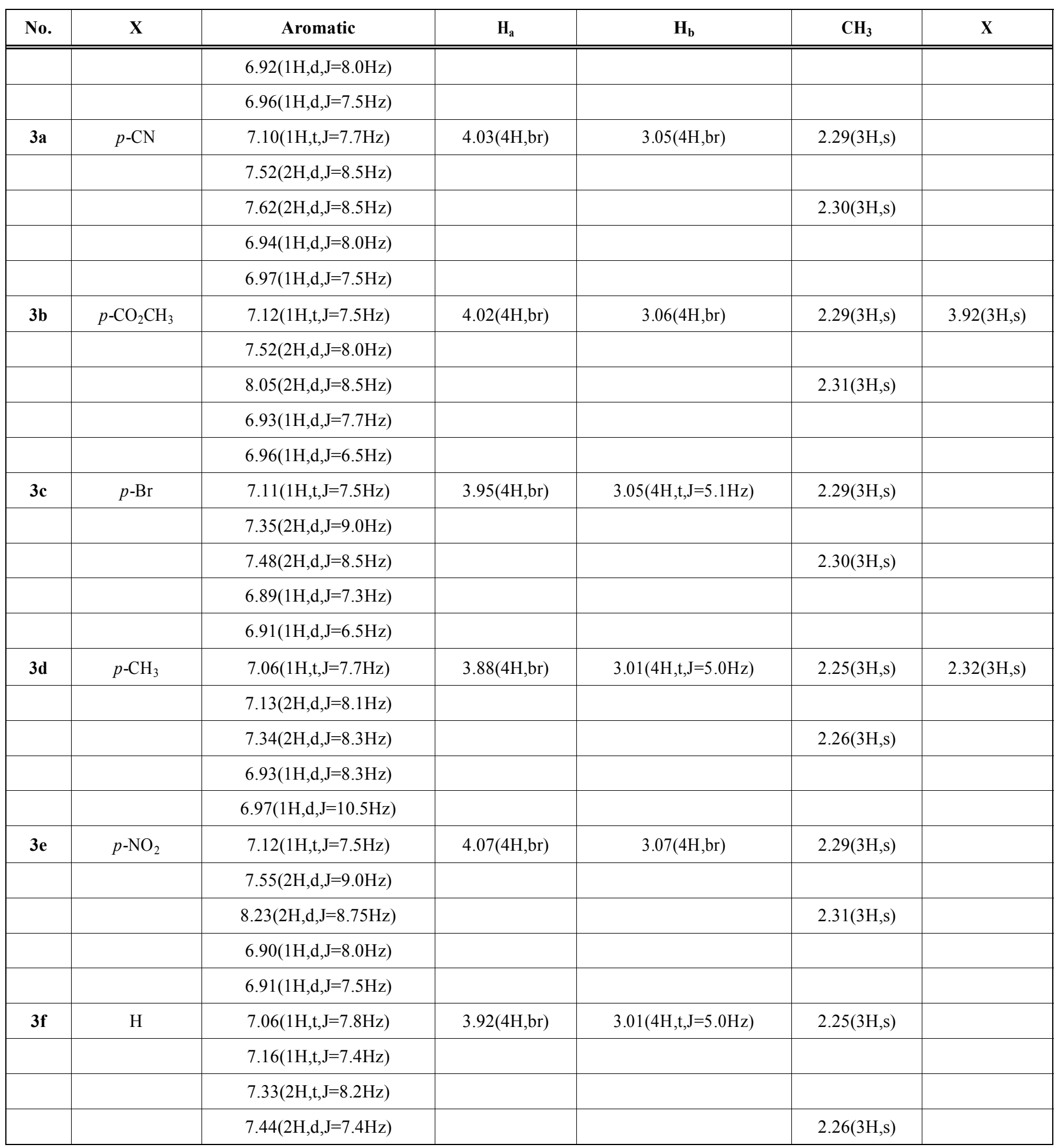

Table 7. ${ }^{1} \mathrm{H}$ NMR Data for $4 a-f$; chemical shifts in ppm relative to $\mathrm{TMS}(1 \%)$ at room temperature in $\mathrm{CDCl}_{3}$.

\begin{tabular}{|c|c|c|c|c|c|c|c|}
\hline No. & $\mathbf{X}$ & Aromatic & $\mathbf{H}_{\mathbf{a}}$ & $\mathbf{H}_{\mathbf{b}}$ & $\mathbf{C H}_{\mathbf{2}}$ & $\mathbf{C H}_{\mathbf{3}}$ & $\mathbf{X}$ \\
\hline \multirow{2}{*}{$4 \mathrm{a}$} & & $7.11(2 \mathrm{H}, \mathrm{d}, \mathrm{J}=7.8 \mathrm{~Hz})$ & & & \\
\cline { 5 - 8 } & $\mathrm{p}-\mathrm{CN}$ & $7.19(2 \mathrm{H}, \mathrm{d}, \mathrm{J}=7.9 \mathrm{~Hz})$ & $3.84(4 \mathrm{H}, \mathrm{br})$ & $2.56(4 \mathrm{H}, \mathrm{br})$ & $3.51(2 \mathrm{H}, \mathrm{s})$ & $2.31(3 \mathrm{H}, \mathrm{s})$ & \\
\cline { 3 - 8 } & & $7.43(2 \mathrm{H}, \mathrm{d}, \mathrm{J}=8.6 \mathrm{~Hz})$ & & & & \\
\hline
\end{tabular}


Table 7. contd.....

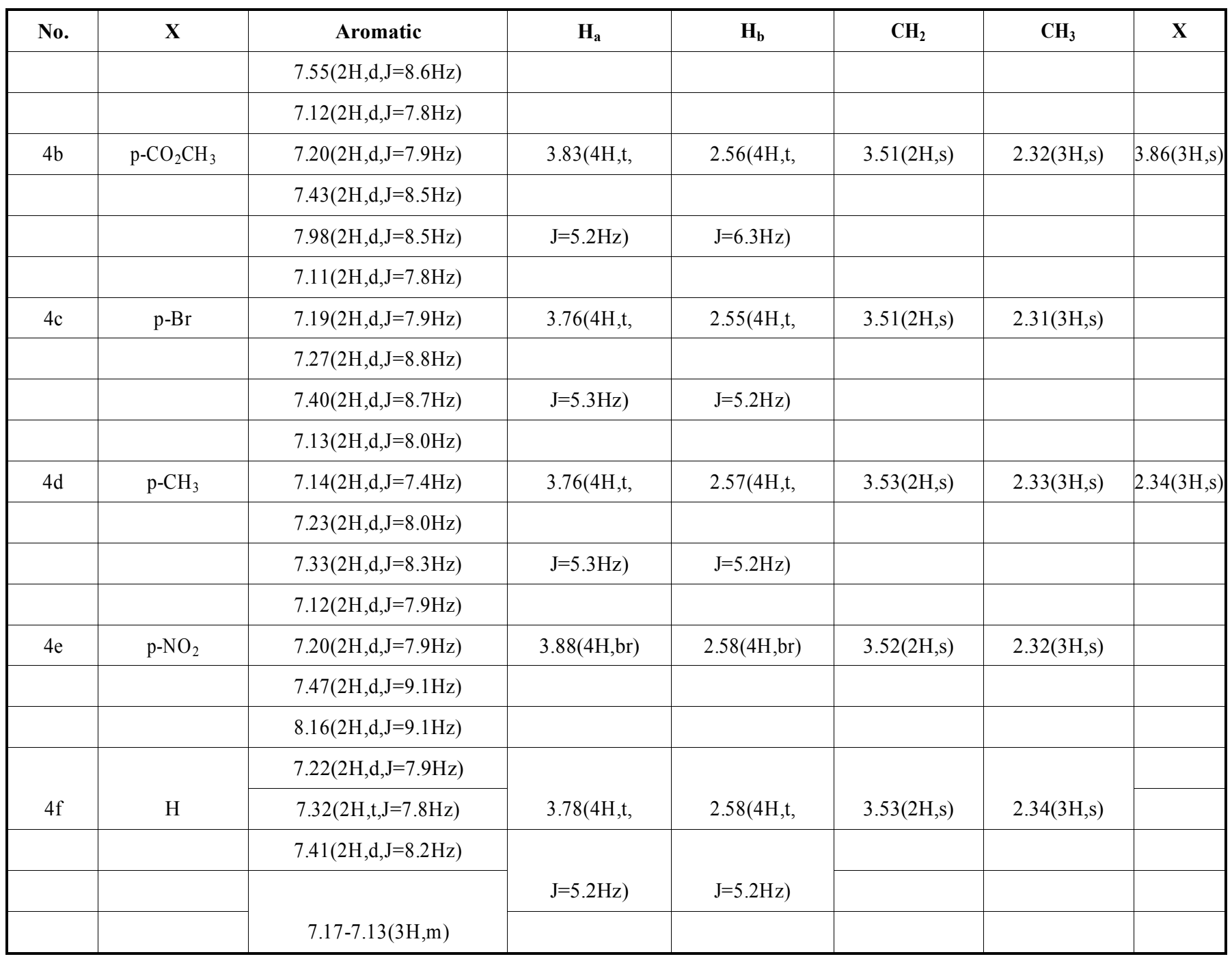

Table 8. ${ }^{1} \mathrm{H}$ NMR Data for $5 \mathrm{a}-\mathrm{e}$; chemical shifts in ppm relative to TMS(1\%) at room temperature in $\mathrm{CDCl}_{3}$.

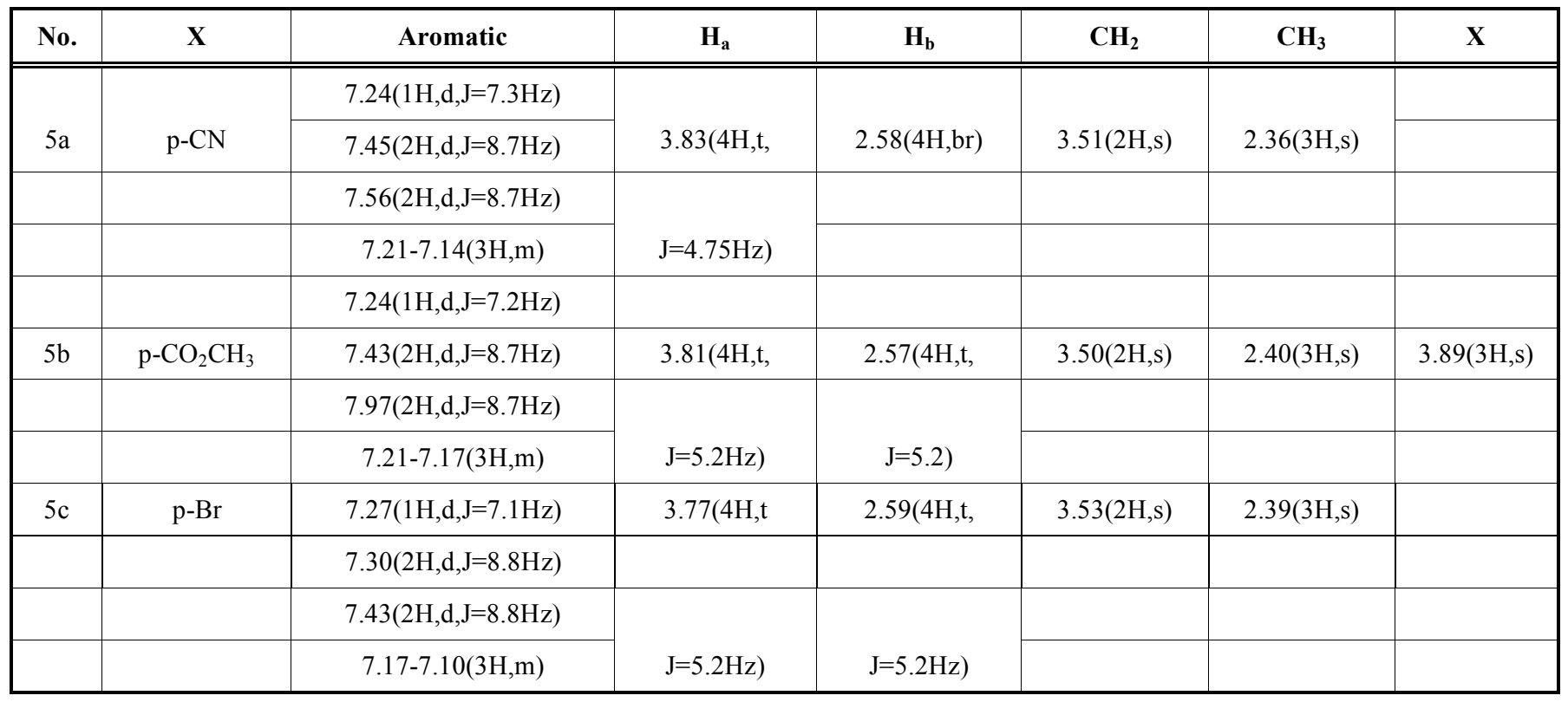


Table 8. contd.....

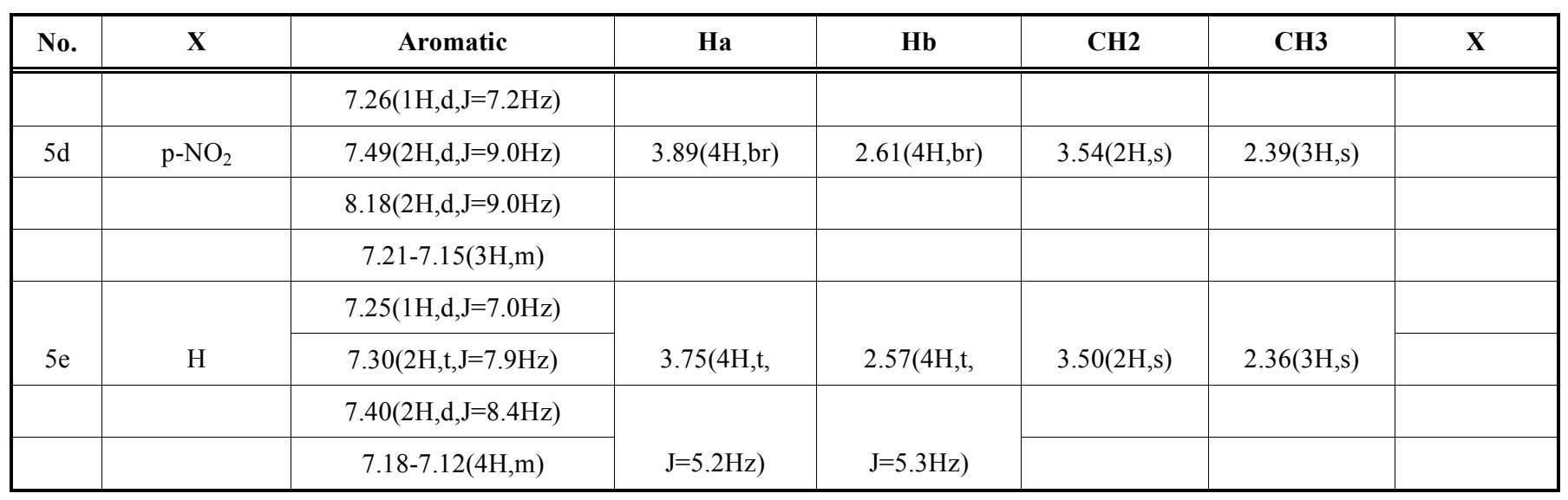

Table 9. ${ }^{1} \mathrm{H}$ NMR Data for 6a-e; chemical shifts in ppm relative to TMS(1\%) at room temperature in $\mathrm{CDCl}_{3}$.

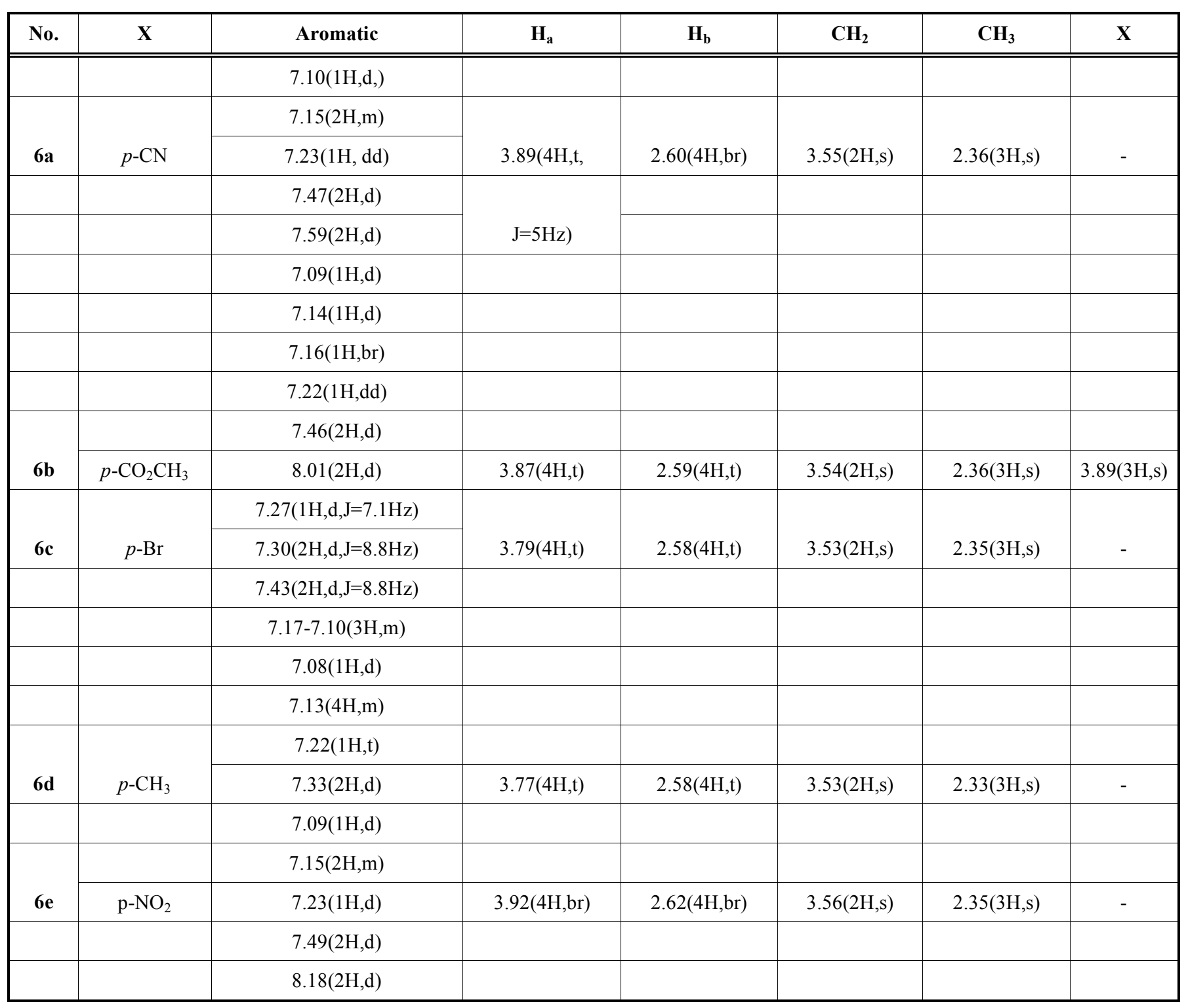


Table 10. ${ }^{1} \mathrm{H}$ NMR Data for $7 \mathrm{a}$; chemical shifts in ppm relative to $\mathrm{TMS}(1 \%)$ at room temperature in $\mathrm{CDCl}_{3}$.

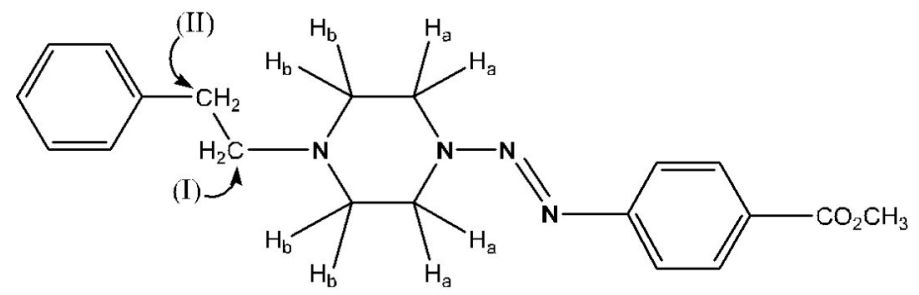

\begin{tabular}{|c|c|c|c|c|c|c|c|}
\hline No. & $\mathbf{X}$ & Aromatic & $\mathbf{H}_{\mathbf{a}}$ & $\mathbf{H}_{\mathbf{b}}$ & $\mathbf{C H}_{\mathbf{2}}(\mathbf{I})$ & $\mathbf{C H}_{\mathbf{2}}(\mathbf{I I})$ & $\mathbf{X}$ \\
\hline \hline & & $8.01(2 \mathrm{H}, \mathrm{d}, \mathrm{J}=8.5 \mathrm{~Hz})$ & $3.89(4 \mathrm{H}, \mathrm{t}$, & & & \\
\hline $\mathbf{7 a}$ & $p-\mathrm{CO}_{2} \mathrm{CH}_{3}$ & $7.46(2 \mathrm{H}, \mathrm{d}, \mathrm{J}=8.5 \mathrm{~Hz})$ & $\mathrm{J}=7.5 \mathrm{~Hz})$ & $2.67(4 \mathrm{H}, \mathrm{br})$ & $2.83(2 \mathrm{H}, \mathrm{t}, \mathrm{J}=8.0 \mathrm{~Hz})$ & $2.68(2 \mathrm{H}, \mathrm{t}, \mathrm{J}=8.0 \mathrm{~Hz})$ & $3.89(3 \mathrm{H}, \mathrm{s})$ \\
\hline & & $7.28(2 \mathrm{H}, \mathrm{t}, \mathrm{J}=7.5 \mathrm{~Hz})$ & & & & \\
\hline & & $7.21(2 \mathrm{H}, \mathrm{d}, \mathrm{J}=7.5 \mathrm{~Hz})$ & & & & & \\
\hline
\end{tabular}

Table 11. ${ }^{13} \mathrm{C}$ NMR Data for 3a-f; chemical shifts in ppm relative to TMS(1\%) at room temperature in $\mathrm{CDCl}_{3}$.

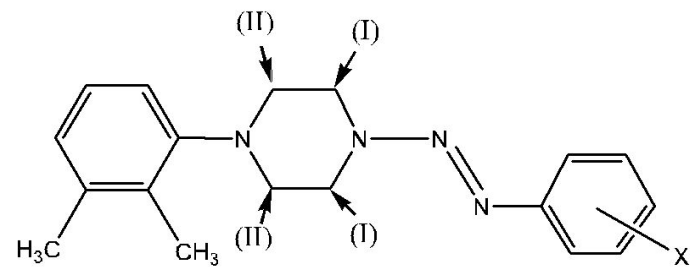

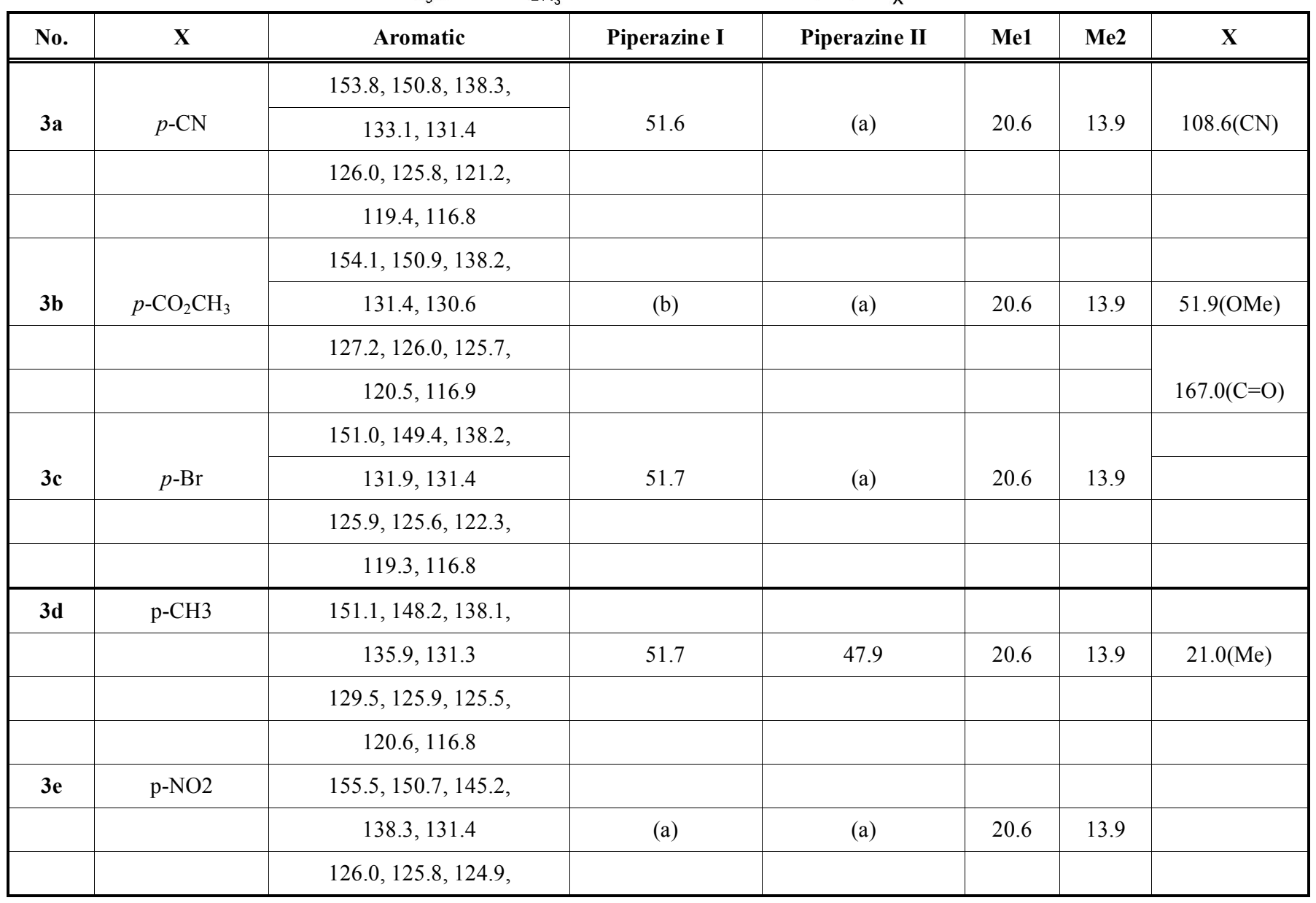


Table 11. Contd.....

\begin{tabular}{|c|c|c|c|c|c|c|c|}
\hline No. & $\mathbf{X}$ & Aromatic & Piperazine I & Piperazine II & Me1 & Me2 & $\mathbf{X}$ \\
\hline & & $120.8,116.9$ & & & & & \\
\hline \multirow[t]{2}{*}{$3 f$} & $\mathrm{H}$ & $130.9,128.5$ & 51.4 & 48.9 & 20.3 & 13.5 & \\
\hline & & $125.8,125.5,125.1$ & & & & & \\
\hline
\end{tabular}

(a) Not detected

(b) Masked by O-methyl carbon signal

Table 12. ${ }^{13} \mathrm{C}$ NMR Data for $4 \mathrm{a}-\mathrm{f}$; chemical shifts in ppm relative to TMS(1\%) at room temperature in $\mathrm{CDCl}_{3}$.

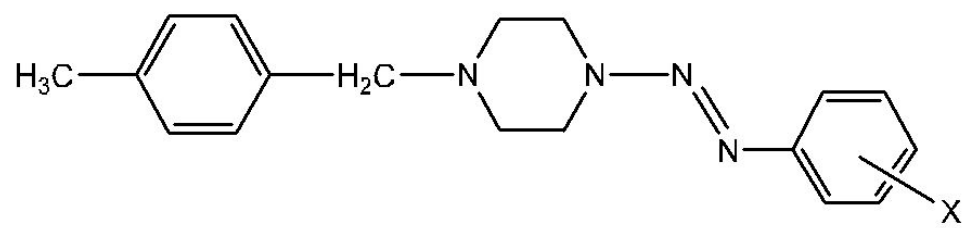

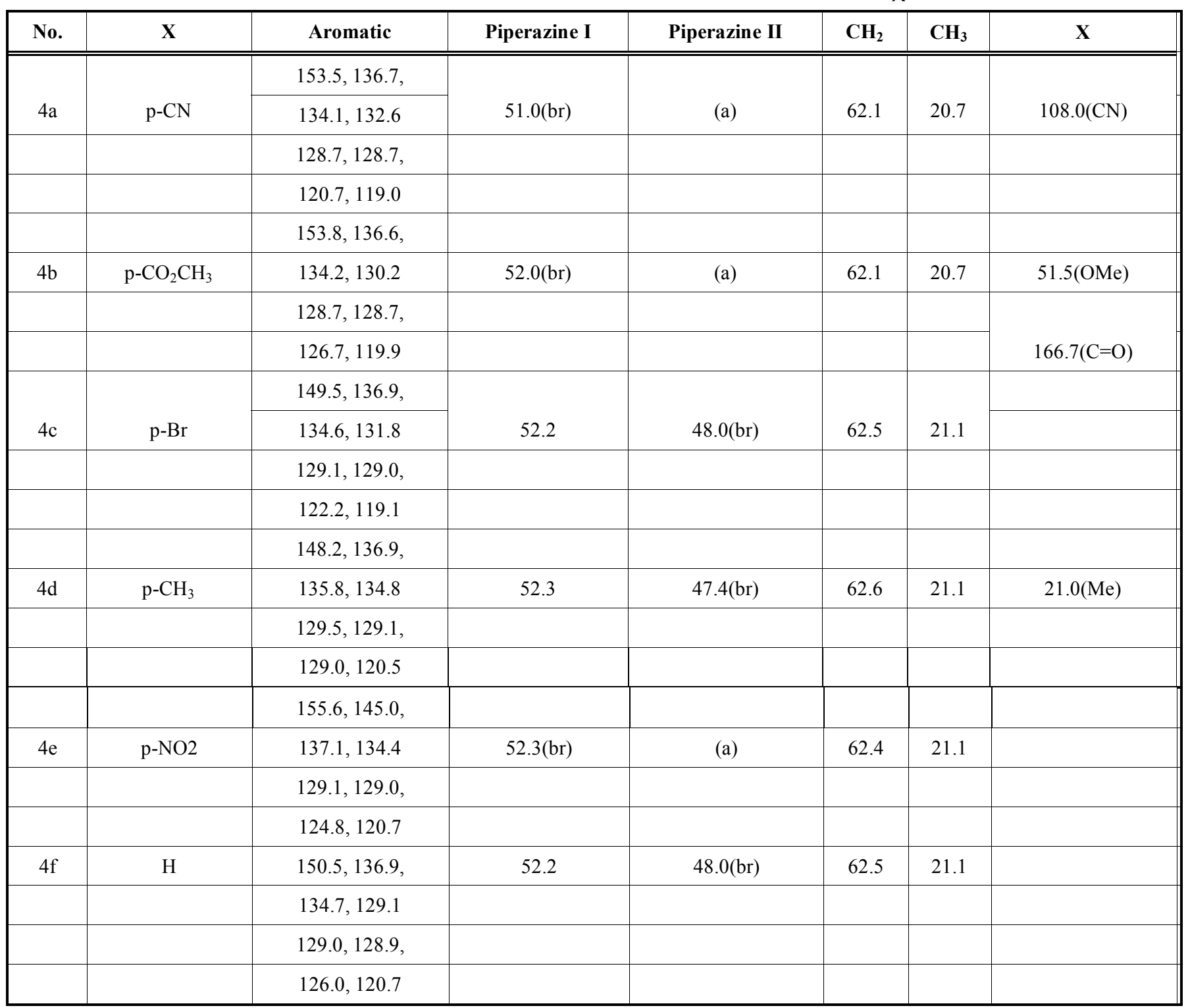

(a) Not detected 
Table 13. ${ }^{13} \mathrm{C}$ NMR Data for $5 \mathrm{a}-\mathrm{e}$; chemical shifts in ppm relative to TMS(1\%) at room temperature in $\mathrm{CDCl}_{3}$.

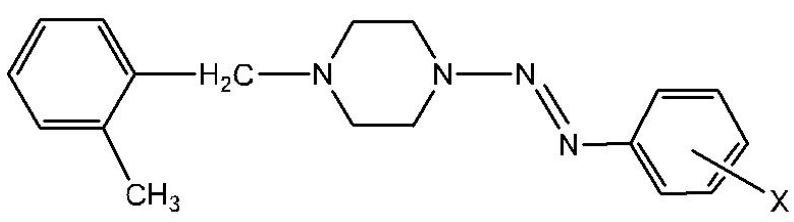

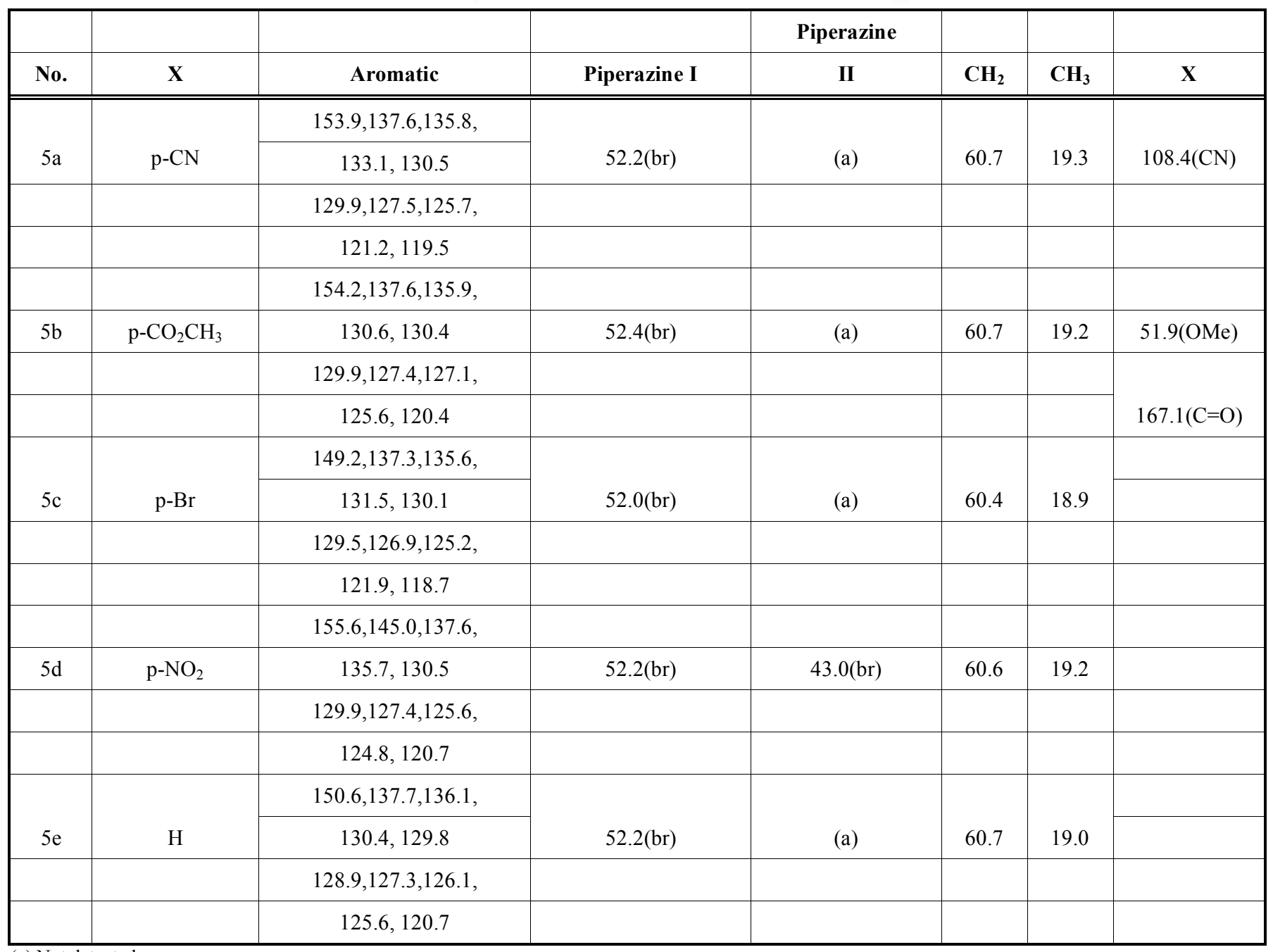

(a) Not detected

Table 14. ${ }^{13} \mathrm{C}$ NMR Data for 6a-e; chemical shifts in ppm relative to $\mathrm{TMS}(1 \%)$ at room temperature in $\mathrm{CDCl}_{3}$.

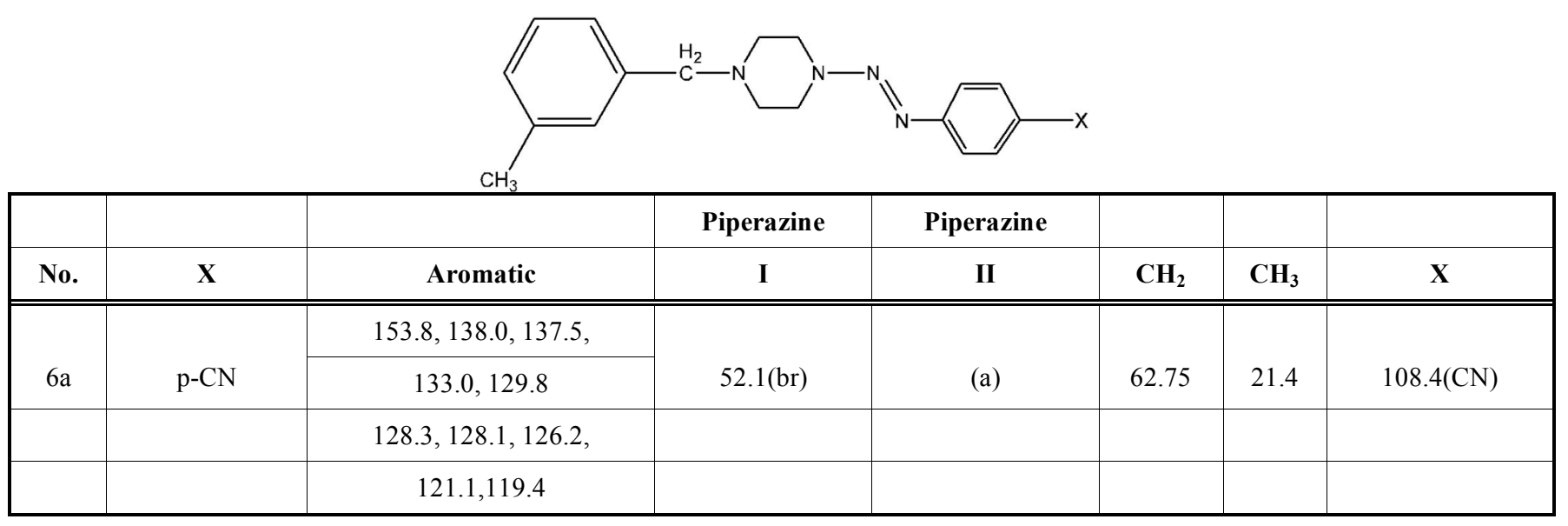


Table 14. Contd.....

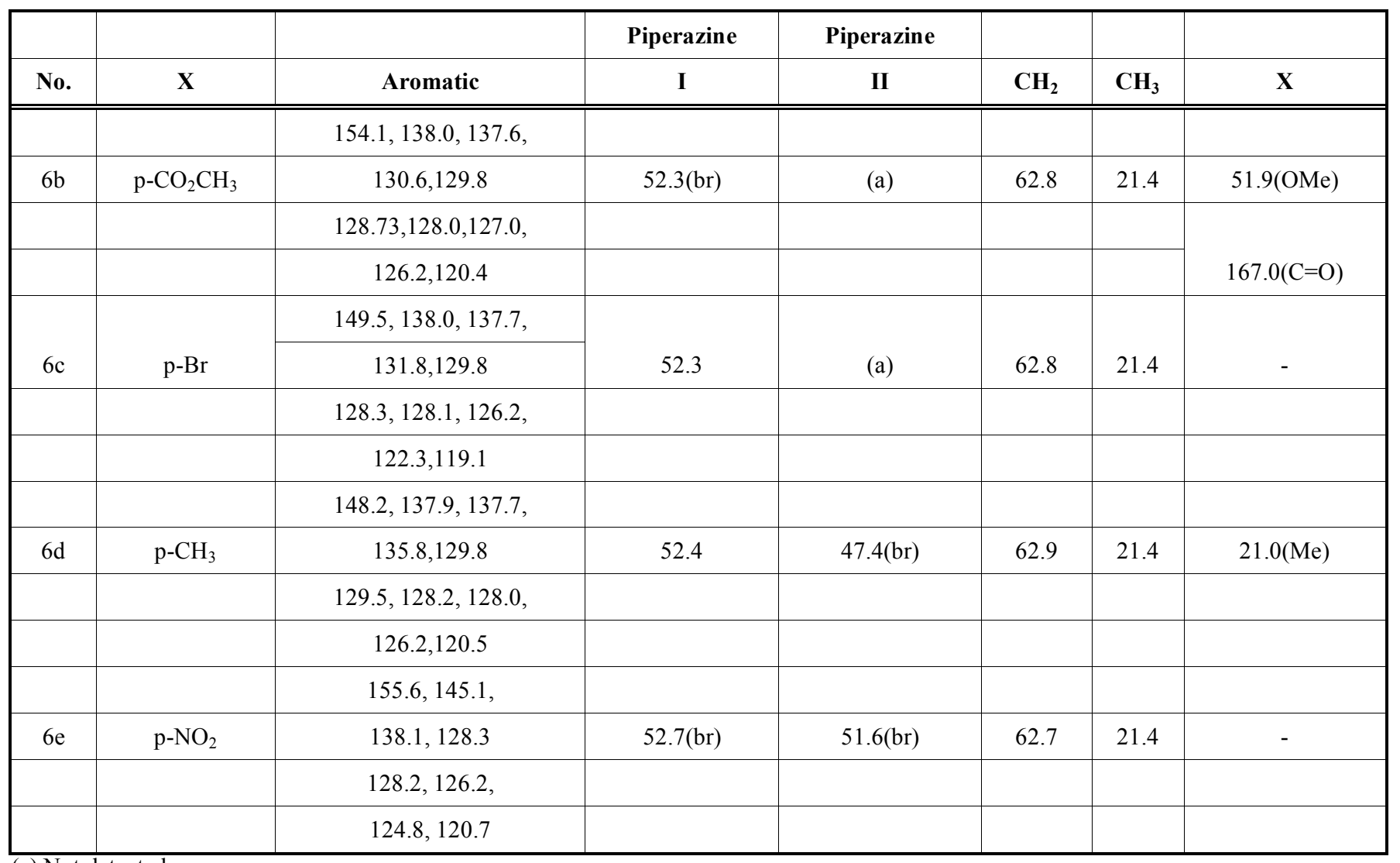

(a) Not detected

Table 15. ${ }^{13} \mathrm{C}$ NMR Data for $7 \mathrm{a}$; chemical shifts in ppm relative to $\mathrm{TMS}(1 \%)$ at room temperature in $\mathrm{CDCl}_{3}$.

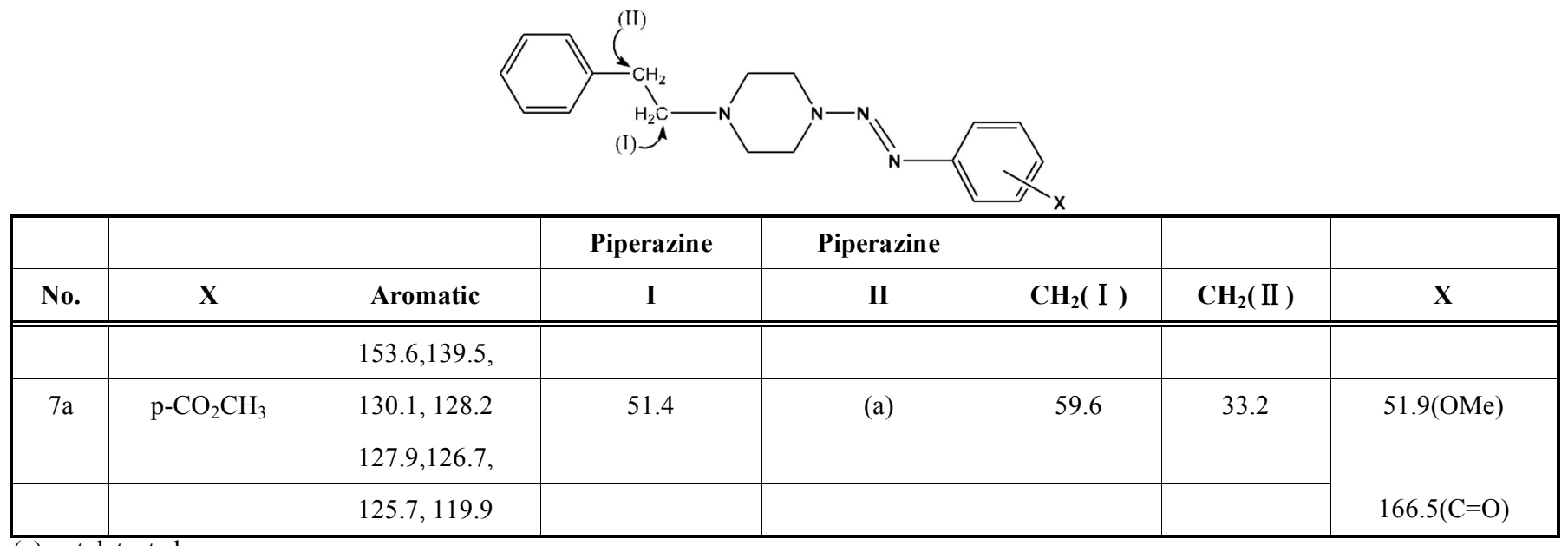

(a) not detected

Table 16. High-resolution electron-ionization mass spectral (EI-MS) data for 3a-f.

\begin{tabular}{|c|c|c|c|c|}
\hline No. & $\mathbf{X}$ & Molecular & Calculated & Experimental \\
\hline & & Formula & Mass & $319.1785 \mathrm{amu}$ \\
\hline$\underline{\mathbf{3 a}}$ & $\mathrm{p}-\mathrm{CN}$ & $\mathrm{C} 19 \mathrm{H} 21 \mathrm{~N} 5$ & $319.1797 \mathrm{amu}$ & \\
\hline$\underline{\mathbf{3 b}}$ & $\mathrm{p}-\mathrm{CO}_{2} \mathrm{Me}$ & $\mathrm{C} 20 \mathrm{H} 24 \mathrm{~N} 4 \mathrm{O} 2$ & $352.1899 \mathrm{amu}$ & $352.1914 \mathrm{amu}$ \\
\hline
\end{tabular}


Table 16. Contd.....

\begin{tabular}{|c|c|c|c|c|}
\hline No. & $\mathbf{X}$ & Molecular & Calculated & Experimental \\
\hline & & Formula & Mass & $372.0957 \mathrm{amu}$ \\
\hline \hline$\underline{\mathbf{3 c}}$ & $\mathrm{p}-\mathrm{Br}$ & $\mathrm{C} 18 \mathrm{H} 21 \mathrm{~N} 4 \mathrm{Br}$ & $372.0949 \mathrm{amu}$ & $308.1994 \mathrm{amu}$ \\
\hline$\underline{\mathbf{3 d}}$ & $\mathrm{p}-\mathrm{CH}_{3}$ & $\mathrm{C} 19 \mathrm{H} 24 \mathrm{~N} 4$ & $308.2001 \mathrm{amu}$ & $339.1702 \mathrm{amu}$ \\
\hline$\underline{\mathbf{3}}$ & $\mathrm{p}-\mathrm{NO}_{2}$ & $\mathrm{C} 18 \mathrm{H} 21 \mathrm{~N} 5 \mathrm{O} 2$ & $294.1844 \mathrm{amu}$ & $294.1830 \mathrm{amu}$ \\
\hline$\underline{\mathbf{3 f}}$ & $\mathrm{H}$ & $\mathrm{C} 18 \mathrm{H} 22 \mathrm{~N} 4$ & & \\
\hline
\end{tabular}

Table 17. High-resolution electron-ionization mass spectral (EI-MS) data for 4a-f.

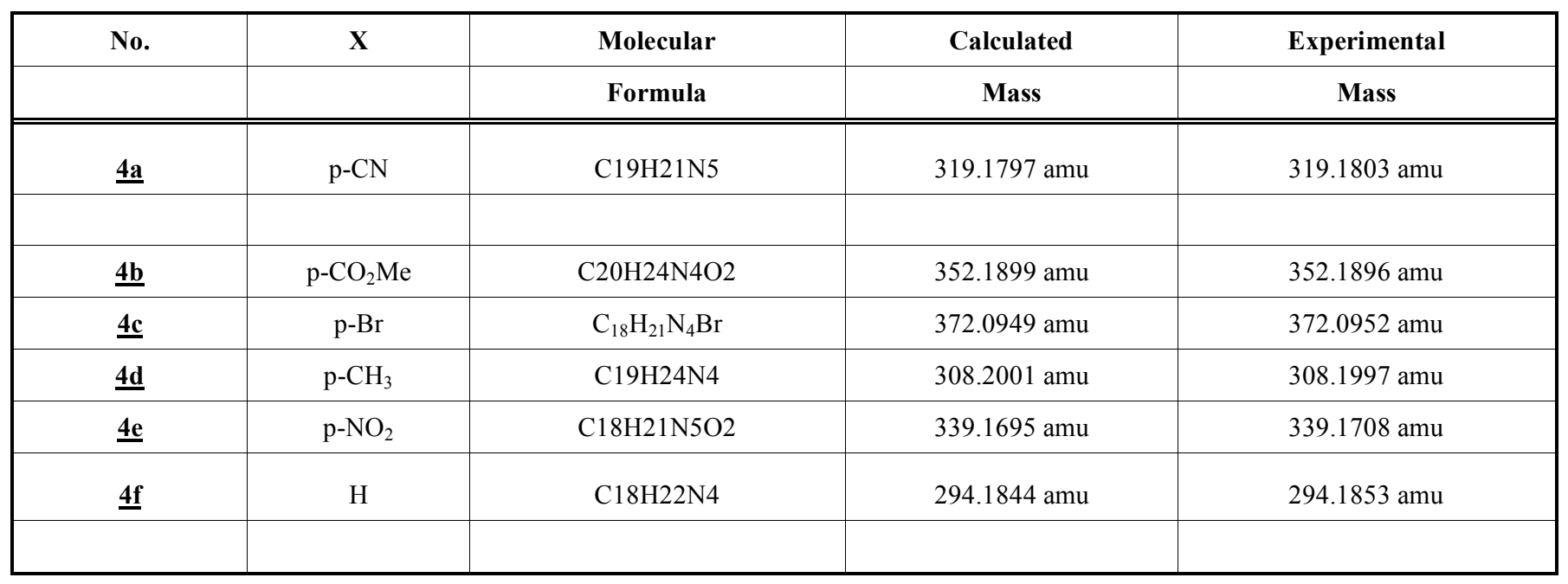

Table 18. High-resolution electron-ionization mass spectral (EI-MS) data for 5a-e.

\begin{tabular}{|c|c|c|c|c|}
\hline No. & $\mathbf{X}$ & Molecular & Calculated & Mass \\
\hline \hline$\underline{\mathbf{a}}$ & & Formula & $319.1797 \mathrm{amu}$ & Mass \\
\hline$\underline{\mathbf{5 b}}$ & $\mathrm{p}-\mathrm{CN}$ & $\mathrm{C} 19 \mathrm{H} 21 \mathrm{~N} 5$ & & $319.1791 \mathrm{amu}$ \\
\hline$\underline{\mathbf{5}}$ & $\mathrm{p}-\mathrm{Cr}$ & $\mathrm{C} 20 \mathrm{H} 24 \mathrm{~N} 4 \mathrm{O} 2$ & $352.1899 \mathrm{amu}$ & $352.1892 \mathrm{amu}$ \\
\hline$\underline{\mathbf{5 d}}$ & $\mathrm{p}-\mathrm{NO}_{2}$ & $\mathrm{C}_{18} \mathrm{H}_{21} \mathrm{~N}_{4} \mathrm{Br}$ & $372.0949 \mathrm{amu}$ & $372.0963 \mathrm{amu}$ \\
\hline$\underline{\mathbf{5 e}}$ & $\mathrm{C} 18 \mathrm{H} 21 \mathrm{~N} 5 \mathrm{O} 2$ & $339.1695 \mathrm{amu}$ & $339.1683 \mathrm{amu}$ \\
\hline
\end{tabular}

Table 19. High-resolution electron-ionization mass spectral (EI-MS) data for 6a-e.

\begin{tabular}{|c|c|c|c|c|}
\hline No. & $\mathbf{X}$ & Molecular & Calculated & Experimental \\
\hline & & Formula & Mass & $320.1857 \mathrm{amu}$ \\
\hline \hline$\underline{\mathbf{6 a}}$ & $\mathrm{p}-\mathrm{CN}$ & $\mathrm{C} 19 \mathrm{H} 22 \mathrm{~N} 5$ & $320.1876 \mathrm{amu}$ & \\
\hline$\underline{\mathbf{6}}$ & & & $375.1797 \mathrm{amu}$ & $375.1791 \mathrm{amu}$ \\
\hline$\underline{\mathbf{6 c}}$ & $\mathrm{p}-\mathrm{CO}_{2} \mathrm{Me}$ & $\mathrm{C}_{20} \mathrm{H}_{24} \mathrm{~N}_{4} \mathrm{O}_{2} \mathrm{Na}$ & $373.1028 \mathrm{amu}$ & $373.1022 \mathrm{amu}$ \\
\hline
\end{tabular}


Table 19. Contd.....

\begin{tabular}{|c|c|c|c|c|}
\hline No. & $\mathbf{X}$ & Molecular & Calculated & Experimental \\
\hline & & Formula & Mass & $331.1893 \mathrm{amu}$ \\
\hline \hline$\underline{\mathbf{6}}$ & $\mathrm{p}-\mathrm{CH}_{3}$ & $\mathrm{C}_{19} \mathrm{H}_{24} \mathrm{~N}_{4} \mathrm{Na}$ & $331.1899 \mathrm{amu}$ & $340.1767 \mathrm{amu}$ \\
\hline$\underline{\mathbf{6 e}}$ & $\mathrm{p}-\mathrm{NO}_{2}$ & $\mathrm{C} 18 \mathrm{H} 22 \mathrm{~N} 5 \mathrm{O} 2$ & $340.1774 \mathrm{amu}$ & \\
\hline
\end{tabular}

Table 20. High-resolution electron-ionization mass spectral (EI-MS) data for 7a.

\begin{tabular}{|c|c|c|c|c|}
\hline No. & $\mathbf{X}$ & Molecular & Calculated & Experimental \\
\hline & & Formula & Mass & Mass \\
\hline \hline$\underline{7 a}$ & $\mathrm{p}-\mathrm{CO}_{2} \mathrm{Me}$ & $\mathrm{C} 20 \mathrm{H} 24 \mathrm{~N} 4 \mathrm{O} 2$ & $352.1899 \mathrm{amu}$ & $352.1890 \mathrm{amu}$ \\
\hline
\end{tabular}

which is attached on the piperazine ring in 3a-f, is a 1,2,3trisubstituted benzene ring system. Predictably this ring system shows two out-of-plane bending vibration modes in the ranges of $719-724 \mathrm{~cm}^{-1}$ and $780-788 \mathrm{~cm}^{-1}$. The aryl group, which is linked to the piperazine ring in $4 \mathbf{a}-\mathbf{f}$, is a para-disubstituted benzene ring, which shows one out-ofplane bending vibration band in the range of 784-801 $\mathrm{cm}^{-1}$. The aryl group, which is connected to the piperazine ring in 5a-e, is an ortho-substituted benzene ring. Compounds 5a-e show one ortho-substituted out-of-plane bending vibration band in the range of 743-746 $\mathrm{cm}^{-1}$. The monosubstituted benzene ring, which joined with the piperazine ring in $7 \mathbf{a}$, shows two out-of-plane bending vibration bands at 699 and $744 \mathrm{~cm}^{-1}$. The carbonyl group of the ester group, which is present in compounds $\mathbf{3 b}, \mathbf{4 b}, \mathbf{5 b}, \mathbf{6 b}$ and $\mathbf{7 a}$, shows a stretching vibration band in the range of $1707-1722 \mathrm{~cm}^{-1}$. In the same compounds, carbon oxygen single bond stretching bands of the ester groups were in the range of 1274-1278 $\mathrm{cm}^{-}$ ${ }^{1}$. Nitrile group stretching vibration bands were observed in 3a, 4a, 5a and 6a in the range of $2218-2225 \mathrm{~cm}^{-1}$. Nitro groups, which were contained in $\mathbf{3 e}, \mathbf{4 e}, \mathbf{5 d}$ and $\mathbf{6 e}$, show symmetric stretching vibration modes in the range of 1341$1379 \mathrm{~cm}^{-1}$ and asymmetric stretching vibration modes in the range of $1507-1509 \mathrm{~cm}^{-1}$.

\section{${ }^{1}$ H NMR Spectroscopic Analysis}

The ${ }^{1} \mathrm{H}$ NMR results are shown in Table $\mathbf{6}$ to Table $\mathbf{1 0 .}$ These results are expressed as chemical shift in ppm relative to $\mathrm{TMS}(1 \%)$ at room temperature in $\mathrm{CDCl}_{3}$. All of the compounds contained two benzene rings; the aromatic signals appeared in the range of $\delta 8.23-6.90$ with a coupling constant $(\mathrm{J})$ in the range of 7.0-9.0 $\mathrm{Hz}$. One of the two benzene rings was initially from aniline derivatives, which are most of the para-substituted anilines except $\mathbf{3 f}, \mathbf{4 f}$, and 5e; such that they show two doublet peaks characteristic of an AA'BB' system in the range of aromatic protons. The highest chemical shift peaks are always due to the two equivalent aromatic protons which are close to the triazene subunit. Some aromatic protons were not resolved due to overlapping peaks in the range of $\delta 7.20-6.90$. The starting material, the aryl piperazine, was studied by NMR spectroscopy as a model compound to distinguish the protons in the two aryl groups, and the protons on the aryl group were matched with the same proton in the final triazene product in an approximate range.

[iii]

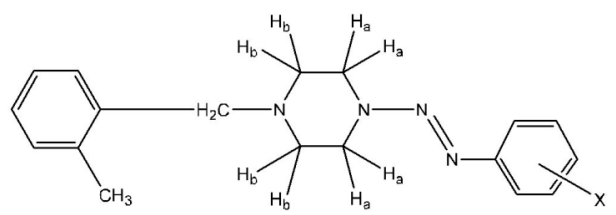

The most significant peaks are the methylene protons on the piperazine ring protons $\mathrm{H}_{\mathrm{a}}$ and $\mathrm{H}_{\mathrm{b}}$ (see schematic [iii] above). As shown in tables 6 to $10, \mathrm{H}_{\mathrm{a}}$ has a higher chemical shift than that of $\mathrm{H}_{\mathrm{b}}$. due to the closer proximity of $\mathrm{H}_{\mathrm{a}}$ to the triazene subunit. In general, the methylene protons are represented by two triplet peaks each integrating for $4 \mathrm{H}$. However, some of the triplet peaks were not resolved resulting in broad peaks, especially for $\mathrm{H}_{\mathrm{a}}$. Such observation was due to the restricted rotation of the nitrogen-nitrogen single bond in the triazene subunit [6]. The $\mathrm{H}_{\mathrm{a}}$ protons have representative peaks in the range of $\delta$ 4.07-3.75 with integration for $4 \mathrm{H}$ and coupling constants in the range of 4.8$5.3 \mathrm{~Hz}$ for the resolving triplet peaks. The $\mathrm{H}_{\mathrm{b}}$ protons have representative peaks in the range of $\delta 3.07-2.55$ with coupling constants in the range of $5.1-6.3 \mathrm{~Hz}$ for the resolving triplet peaks. Signals arising from the substituents on the benzene ring are consistent with the substituents present, such as the O-methyl protons arising as singlets in the range $\delta 3.08-3.92$ in the spectra of $\mathbf{3 b}, \mathbf{4 b}, \mathbf{5 b}, \mathbf{6 b}$ and $\mathbf{7 a}$. The ethylene bridge linking the piperazine and aryl rings in compound $7 \mathrm{a}$ is manifested by two $2 \mathrm{H}$ triplet peaks at $\delta 2.68$ ppm and $\delta 2.83 \mathrm{ppm}$ with a coupling constant $\mathrm{J}=8.0 \mathrm{~Hz}$. The full analysis of the ${ }^{1} \mathrm{H}$ spectrum of $\mathbf{7 a}$ was complicated by the coincidence of the signals of $\mathrm{H}^{\mathrm{a}}$ and the O-methyl protons at $3.89 \mathrm{ppm}$ and the (considerable overlap of the triplets of $\mathrm{CH}_{2}$ (II) and proton $\mathrm{H}^{\mathrm{b}}$.

\section{${ }^{13}$ C NMR}

The structures of all new compounds have been investigated by ${ }^{13} \mathrm{C}$ NMR spectroscopy (see Tables $\mathbf{1 1}$ to $\mathbf{1 5}$ ). For compounds 3a-f, there are ten magnetically nonequivalent aromatic carbon atoms in each molecule. The aromatic carbons resonate in the range of $\delta 116-155 \mathrm{ppm}$. The mono- or para-substituted benzene ring has four 
(iv)

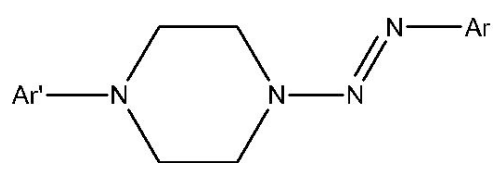

magnetically non-equivalent carbon atoms, since two carbon atoms on the ortho position are magnetically equivalent and the same goes for the two carbon atoms on the meta position. The 1,2,3-trisubstituted benzene ring has six carbon atoms that are all magnetically non-equivalent. The carbon atoms in the piperazine ring were often difficult to resolve due to the dynamic equilibrium in the triazene moiety. The carbon atoms in the piperazine ring are represented by the two peaks (both are usually broad peaks, if observed) in the range of $\delta$ 52.4-43.0 ppm. The two methyl groups on the 1,2,3trisubstituted benzene ring were represented by the two peaks in the ranges of $\delta 20.6-20.3 \mathrm{ppm}$ and $\delta 13.9-13.5 \mathrm{ppm}$. The nitrile group carbons resonate at $\delta 108.6 \mathrm{ppm}$. The carbonyl carbon in the ester subunit was represented by a peak at $\delta 167.0 \mathrm{ppm}$; the methyl group in the ester group was represented by a peak at $\delta 51.9 \mathrm{ppm}$. In 3d, the p-tolyl methyl group resonates at $\delta 21.0 \mathrm{ppm}$.

For the compounds $\mathbf{4 a - f}$, there are two benzene rings, one mono-substituted and one para-disubstituted. Therefore, there are eight magnetically non-equivalent aromatic carbons in each compound, which are represented by eight peaks in the range of $\delta 155.6-119.0 \mathrm{ppm}$. For the piperazine carbons and the substituents on the aniline derivatives, chemical shifts similar to those seen previously in compounds 3a-f were observed. The two carbons linked to the aryl group, in which the aryl group is attached to the piperazine ring, is represented by two peaks in the ranges of $\delta$ 62.6-62.1 ppm and $\delta 21.1-20.7 \mathrm{ppm}$. The carbons of the methylene groups between the benzene ring and the piperazine ring resonate in higher frequency $(\delta 62.6-62.1 \mathrm{ppm})$.

For the compounds 5a-e, there are ten magnetically nonequivalent aromatic carbons for each compound, which include six carbons from the ortho substituted benzene ring and four carbons from the para- or mono-substituted benzene ring. The ten aromatic carbons resonated in the range of $\delta$ 155.6-118.7 ppm. All other carbons located in the piperazine ring and the substituents have similar chemical shifts as described previously for compounds 3a-f.

For the compounds 6a-e, there are ten magnetically nonequivalent aromatic carbons for each compound, which include six carbons from the meta substituted benzene ring and four carbons from the para- or mono-substituted benzene ring. The ten aromatic carbons resonated in the range of $\delta$ 155.6-118.7 ppm. All other carbons located in the piperazine ring and the substituents have similar chemical shifts as described previously for compounds 3a-f.

For the compound $\mathbf{7 a}$, eight magnetically non-equivalent aromatic carbons were involved in each compound, due to the fact that the two benzene rings were para- and monosubstituted rings. The two methylene groups between the benzene ring and the piperazine ring were represented by two peaks at $\delta 59.6$ and $\delta 51.4 \mathrm{ppm}$. The higher chemical shift accounts for the methylene group linked to the piperazine ring. All the other carbons in the molecule

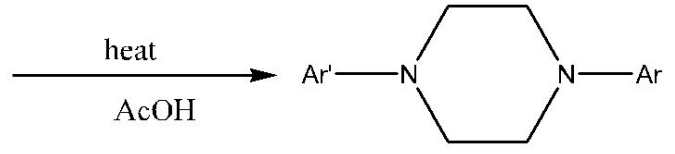

resonated in the reasonable range as described previously for compounds 3a-f.

\section{MASS SPECTRAL ANALYSIS}

The high resolution mass spectrometry (MS) results are shown for all new compounds in Tables 16 to 20. Mass spectroscopy (MS) is an important tool in synthetic organic chemistry, especially high resolution MS. Although MS can be used to predict the conformation of the molecule by matching the peaks to the small fragments of the molecule, the MS results can not be used because it is not capable of distinguishing the isomers in this project. The main use of the MS is to provide the evidence of the formation of the target molecule by matching the actual molecular weight with the experimental molecular ion mass. In the high resolution MS, the results were obtained with the standard deviation of $+/-0.0008 \mathrm{amu}$. According to the MS results, all the molecular ions have been found in the spectra and matched with the actual mass of the molecule within the standard deviation.

\section{CONCLUSION}

The series of isomers of N-(2,3-dimethylphenyl)-N'(aryldiazenyl)-piperazines were prepared in this study. The physical data of all the compounds were measured. All the compounds were extensively characterized through infrared, $\mathrm{NMR}$, and mass spectral analysis. It is always interesting to speculate on the potential applications of new compounds like those reported in this paper. The new 1-aryl-4[aryldiazenyl]-piperazines $(\underline{\mathbf{2} \mathbf{d}})$ have the potential to undergo thermal cleavage under appropriate conditions to provide a route to unsymmetrically substituted N-aryl-N'arylpiperazines (ㅇ). In a previous report [7], it was shown that the synmmetrical 1,4-bis-(2-aryldiazen-1-yl-)piperazines (1) undergo thermal cleavage in acetic acid to afford symmetrical N,N'-diarylpiperazines. The analogous reaction of $\underline{\mathbf{2 d}}$ would afford the unsymmetricaly substituted piperazines $(\underline{\mathbf{8}})$ thus:

Further work in this laboratory will be undertaken to try to make this idea a reality.

Aryldiazenylpiperazines have also been utilized as a means to the immobilization of a diazonium ion by covalent linkage to piperazine attached to a (Merrifield-)resin. The resin bound 1-aryldiazenyl)piperazine was used as a substrate for a Wallach reaction with hydrogen $[18 \mathrm{~F}]$ fluoride to produce radio-labelled 2-fluorophenyl phenyl ether [8].

\section{CONFLICT OF INTEREST}

The authors confirm that this article content has no conflict of interest.

\section{ACKNOWLEDGEMENTS}

The authors are grateful to the Natural Sciences and Engineering Research Council of Canada (NSERC) for a 
Discovery Grant to the principal author $(\mathrm{KV})$. We are also grateful to the Faculty of Graduate Studies and Research at Saint Mary's University for a Summer Research Award to Chenguang Fan. We are also grateful to the Atlantic Region Magnetic Resonance Centre at Dalhousie University for providing NMR spectra, and to Dalhousie University for providing mass spectral data. In particular, we would like to thank Dr. Mike Lumsden for assistance with the NMR spectral data, and Mr. Xiao Feng for assistance with mass spectra.

\section{REFERENCES}

[1] Little, V. R.; Tingley, R.; Vaughan, K. Triazene derivatives of $[1, \mathrm{x}]$-diaza-cycloalkanes. Part III. Synthesis and characterization of a series of 1,4-di[2-aryl-1-diazenyl]piperazines. Can. J. Chem., 2005, 83, 471-476;

[2] Hunter, N.; Vaughan, K. Triazene derivatives of $[1, \mathrm{x}]-$ diazacycloalkanes. Part IX. Synthesis and characterization of a series of 1,4-di[2-aryl-1-diazenyl]-2,6-dimethylpiperazines. Can. J. Chem., 2010, $88,344-351$.

[3] Little, V. R.; Vaughan, K. Synthesis and Characterization of a series of 1-methyl-4-[2-aryl-1-diazenyl]piperazines and a series of ethyl 4-[2-aryl-1-diazenyl]-1-piperazinecarboxylates. Can. J. Chem., 2004, 82, 1294-1303.

[4] MacLeod, E.; Vaughan, K. Synthesis and characterization of new compounds in the series 1-alkyl-4-[2-aryl-1-diazenyl]piperazines. Open Org. Chem. J., 2015, 9, 1-8.

[5] Little, V. R.; Vaughan, K. Synthesis and Characterization of Several Series of 4-Acyl-1-[2-aryl-1-diazenyl]piperazines. Can. J. Chem., 2014, 92, 838-848.

[6] Barra, M.; Srivastava, S.; Brockman, E. Substituent and solvent effects on the N2-N3 hindered rotation of cis-1,3diphenyltriazenes. J. Phys. Org. Chem., 2004, 17, 1057- 1060.

[7] Yanarates, E.; Disli, A.; Yildirir, Y. New N,N'-bis(substitutedphenylazo)-piperazines and their cleavage reactions in acetic acid. Org. Prep. Proced. Int., 1999, 31, 429-433.

[8] Riss, P. J.; Kuschel, S.; Aigbirhio. No carrier added nucleophilic aromatic radiofluorination using solid phase supported arene diazonium sulfonates and 10(aryldiazenyl-)piperazines. Tetrahedron. Lett., 2012, 53(14), 1717-1719.

(C) Fan and Vaughan; Licensee Bentham Open.

This is an open access article licensed under the terms of the (https://creativecommons.org/licenses/by/4.0/legalcode), which permits unrestricted, noncommercial use, distribution and reproduction in any medium, provided the work is properly cited. 